\title{
Relação entre índice de massa corporal e escala de avaliação da síndrome positiva e negativa em portadores de esquizofrenia atendidos em um serviço especializado
}

\section{Relationship between body mass index and positive and negative syndrome assessment scale in schizophrenia patients served at a specialized service}

Juliana Raissa Oliveira Ricarte ${ }^{1,2}$. Joseline Maria Alves Gomes Recamonde ${ }^{2,3}$. Natasha Farias Pitts ${ }^{1,2}$. Ana Patrícia Nogueira Aguiar ${ }^{1,2}$. Rayanne Silva Vieira Lima ${ }^{1,2}$. Fábio Gomes de Matos e Souza ${ }^{1,2}$. Priscila da Silva Mendonça $a^{2,3}$.

1 Universidade Federal do Ceará (UFC), Fortaleza, Ceará, Brasil. 2 Hospital Universitário Walter Cantídio (HUWC), Fortaleza, Ceará, Brasil. 3 Empresa Brasileira de Serviços Hospitalares (EBSERH), Fortaleza, Ceará, Brasil.

\section{RESUMO}

Objetivo: avaliar o estado nutricional e relação com o grau da sintomatologia através da Escala de Avaliação da Síndrome Positiva e Negativa (PANSS) em esquizofrênicos atendidos em um serviço especializado em Fortaleza, Ceará, Brasil. Metodologia: foram avaliados 154 pacientes acompanhados em um ambulatório de psiquiatria, com serviço especializado no tratamento de esquizofrenia de um hospital universitário, situado em Fortaleza, Ceará. As variáveis estudadas foram: peso, altura, IMC, presença de comorbidades, uso prévio de substâncias psicoativas, antipsicóticos e antidepressivos e PANSS. Resultados: a amostra constituiu-se principalmente por homens $(58,4 \%)$. A prevalência de comorbidades foi de 29,9\%. Observou-se maior incidência de: dislipidemias (17,5\%) e hipertensão arterial sistêmica (11,0\%). Verificou-se que 44,8\% dos pacientes já fizeram uso de substâncias psicoativas por pelo menos uma vez; 50,6\% utilizaram antidepressivos e 96,1\% utilizaram antipsicóticos antes de chegar ao serviço especializado. De acordo com o IMC, 65,58\% dos pacientes apresentavam excesso de peso, 31,81\% eram eutróficos e $2,59 \%$ tinham baixo peso. Houve uma correlação negativa entre IMC e PANSS positiva $(r=-0,171 ; p=0,043)$ e uma associação entre sobrepeso e diabetes $(p=0,023)$ e sobrepeso e dislipidemia $(p=0,030)$. Conclusão: pacientes esquizofrênicos constituem um grupo de risco nutricional. Portanto, evidencia-se a importância do cuidado nutricional em todos os estágios do tratamento.

Palavras-chave: Avaliação nutricional. Esquizofrenia. Saúde mental.

\section{ABSTRACT}

Objective: Evaluate the relationship between body mass index (BMI) and degree of symptoms by Positive and Negative Syndrome Scale (PANSS) in schizophrenic attended in a specialized service in Fortaleza, Ceará, Brazil. Methodology: We evaluated 154 patients attending a psychiatric outpatient clinic at a University Hospital in Fortaleza, Ceará, Brazil. The variables studied were: weight, height, BMI, presence of comorbidities, previous use of psychoactive substances, antipsychotics and antidepressants and PANSS. Results: The sample consisted mostly in men, 58,4\%. The prevalence of comorbidities was $29,9 \%$ among patients. Those of higher incidence were dyslipidemia (17,5\%) and hypertension (SAH) (11,0\%). It was verified that 44,8\% of patients had a history of lifetime substance use; 50,6\% had use antidepressants and 96,1\% used antipsychotics before arriving at the service specialist. According to the BMI 65,58\% of patients had excess weight, 31,81\% were eutrophic and 2,59\% had low weight. There was negative correlation significant between BMI and PANSS positive $(r=-0,171 ; p=0,043)$. It was found association between overweight and diabetes $(\mathrm{p}=0,023)$; and overweight and dyslipidemia $(\mathrm{p}=0,030)$. Conclusion: We can observe that schizophrenic patients constitute a group of nutritional risk. Therefore, it is evident the importance of nutritional care at all stages of treatment.

Keywords: Nutrition assessment. Schizophrenia. Mental health.

Autor correspondente: Juliana Raissa Oliveira Ricarte, Rua José Justa, 4308, São João do Tauape, Fortaleza, Ceará. CEP: 60120-290. Telefone: +55 85 99807-9677. E-mail: juliana_raissa@outlook.com

Conflito de interesses: Não há qualquer conflito de interesses por parte de qualquer um dos autores.

Recebido em: 13 Nov 2019; Revisado em: 03 Out 2020; Aceito em: 12 Nov 2021. 


\section{INTRODUÇÃO}

A esquizofrenia é um grave transtorno mental diagnosticado através da presença de sintomas graves, que são classificados em sintomas positivos (delírios, alucinações e discurso desorganizado) e sintomas negativos (embotamento afetivo, déficit de pensamento e avolia). Os pacientes podem apresentar comportamento inadequado, humor disfórico, alterações do ciclo circadiano, falta de interesse na alimentação e recusa da comida. $^{1}$

Além disso, essa condição psiquiátrica tem profundo impacto sobre relacionamentos interpessoais, estilo de vida, autocuidado, em especial sobre o comportamento alimentar. É comum observar nesses indivíduos o aumento excessivo do apetite e do peso corporal, assim como a redução do apetite e perda de peso de forma intencional ou não. ${ }^{2}$

Estudos prévios mostram elevada prevalência de sobrepeso, obesidade e efeitos colaterais ao longo do tratamento com antipsicóticos. Esses efeitos estão associados ao ganho de peso, aumento da circunferência abdominal, distúrbios no metabolismo de glicose e lipídeos, elevação da pressão arterial sistêmica e doenças cardiovasculares. ${ }^{3}$

Nesse sentido, a maior parte das pesquisas realizadas nos últimos anos com pacientes esquizofrênicos se concentram no estudo da obesidade e dos distúrbios metabólicos. ${ }^{4}$ Sendo assim, os estudos que avaliam desnutrição ou baixa ingestão alimentar em meio a sintomas comuns como delírios e alucinações agudas são escassos. Uma forma de avaliar essa sintomatologia é por meio da Escala da Síndrome Positiva e Negativa (PANSS), que viabiliza examinar os sintomas negativos e positivos, a psicopatologia geral e as mudanças geradas pelo uso dos fármacos. Trata-se de uma ferramenta amplamente utilizada, principalmente em estudos clínicos, que descreve a condição psicopatológica do paciente e quantifica o efeito do tratamento. ${ }^{5}$

Tendo em vista que os pacientes esquizofrênicos com sintomas positivos exacerbados compõem um grupo de risco para redução do consumo alimentar resultando diversas vezes em diminuição do peso corporal, além da escassez de estudos que relacionem aspectos nutricionais com a sintomatologia da doença, este estudo tem como objetivo avaliar o estado nutricional e relação com o grau da sintomatologia através da PANSS em esquizofrênicos atendidos em um serviço especializado em Fortaleza, Ceará, Brasil.

\section{METODOLOGIA}

Trata-se de um estudo transversal, de natureza quantitativa. A pesquisa foi realizada em um ambulatório de psiquiatria, com serviço especializado no tratamento de esquizofrenia de um Hospital Universitário, situado em Fortaleza, Ceará.

Foram analisados os prontuários com registros de avaliação inicial de 154 pacientes, com idade igual ou superior a dezoito anos, de ambos os sexos, diagnosticados com esquizofrenia, atendidos entre os anos 2011 a 2017. Foram excluídos os pacientes com registros incompletos e os pacientes diagnosticados com outro transtorno mental. O estudo foi submetido e aprovado pelo Comitê de Ética e Pesquisa do Hospital Universitário Walter Cantídio, parecer $\mathrm{n}^{\mathrm{o}}: 2.357 .286$.

As variáveis estudadas foram: peso, altura, Índice de Massa Corporal (IMC), presença de comorbidades (diabetes, hipertensão arterial sistêmica, dislipidemia, hipertireoidismo), uso prévio de substancias psicoativas (álcool, tabaco, maconha, crack, cocaína, zabumba e santo daime), de antipsicóticos (típicos e atípicos) e de antidepressivos e análise da PANSS.

As análises estatísticas foram realizadas no Software Statistical Package for the Social Sciences (SPSS ${ }^{\circledR}$ ), versão 19.0. A verificação de normalidade foi realizada através do Teste Kolmogorov-Smirnov. As variáveis discretas foram analisadas através do teste qui-quadrado $\left(\chi^{2}\right)$ ou teste Exato de Fisher e submetidas à análise de risco através de Regressão Logística Multinominal, expressa em Odds Ratio (OR). As variáveis contínuas foram submetidas à análise de Correlação de Pearson (r). O nível de significância foi fixado em $p<0,05$.

\section{RESULTADOS}

Dos 154 pacientes avaliados, 58,4\% $(\mathrm{n}=90)$ eram do sexo masculino. A média de idade foi de 44,29 $\pm 14,32$ anos. Dos pacientes que chegaram ao serviço, 29,9\% $(n=46)$ apresentavam alguma comorbidade. Destas, as de maior prevalência foram dislipidemia, observada em 17,5\% ( $\mathrm{n}=$ 27) e hipertensão arterial sistêmica (HAS) em 11,0\% (n=17) dos pacientes. Verificou-se que 44,8\% $(n=69)$ dos pacientes faziam uso de substância psicoativa. Em relação ao uso de medicações, 50,6\% $(\mathrm{n}=78)$ tinham feito uso de algum antidepressivo previamente e $96,1 \%(n=148)$ utilizavam antipsicóticos antes de chegar ao serviço especializado. Destes, $72,7 \%(\mathrm{n}=112)$ faziam uso dos neurolépticos típicos e $96,8 \%(n=149)$ faziam uso dos atípicos no momento da triagem. Segundo a classificação do IMC, 65,58\% dos pacientes apresentavam excesso de peso, $31,81 \%$ eutrofia e $2,59 \%$ baixo peso. Quanto a classificação do nível da PANSS, $67,5 \%(\mathrm{n}=104)$ dos pacientes apresentaram PANSS dentro de um nível considerado estável, enquanto 26,0\% $(\mathrm{n}=40)$ exibiram PANSS com algum sintoma exacerbado (Tabela 01).

Houve correlação negativa significativa entre baixo IMC e PANSS positiva $(r=-0,171 ; p=0,043)$, no entanto, não houve correlação significativa entre IMC geral e PANSS negativa $(r$ $=-0,155$ e $p=0,068)$. Não houve associação significativa entre as classificações de IMC $\left(<25 \mathrm{~kg} / \mathrm{m}^{2}\right.$ e $\left.\geq 25 \mathrm{~kg} / \mathrm{m}^{2}\right)$ e as classificações da PANSS, o uso prévio de diversas substâncias psicoativas, de antidepressivos e de antipsicóticos (Tabela 1). Pacientes com IMC $\geq 25 \mathrm{~kg} / \mathrm{m}^{2}$ tiveram significativamente mais diabetes $(\mathrm{p}=0,023 ; \mathrm{OR}=1,106 ; \mathrm{IC}=[1,039 ; 1,178]) \mathrm{e}$ dislipidemia $(p=0,030 ; \mathrm{OR}=3,265 ; \mathrm{IC}=[1,054 ; 10,026])$ (Tabela 2). 
Tabela 1. Associação entre IMC, PANSS, uso de substâncias psicoativas, antipsicóticos e antidepressivos em esquizofrênicos atendidos em um ambulatório especializado, entre os anos de 2011 a 2017, Fortaleza, Ceará.

\begin{tabular}{|c|c|c|c|}
\hline \multirow{3}{*}{ Características } & \multicolumn{2}{|c|}{$I M C(n / \%)$} & \multirow{3}{*}{ p valor } \\
\hline & $<25 \mathrm{Kg} / \mathrm{m}^{2}$ & $\geq 25 \mathrm{Kg} / \mathrm{m}^{2}$ & \\
\hline & $\mathrm{n}=50(32,5)$ & $\mathrm{n}=104(67,5)$ & \\
\hline \multicolumn{4}{|l|}{ Sexo } \\
\hline Masculino & $26(52,0)$ & $64(61,5)$ & \multirow{2}{*}{0,261} \\
\hline Feminino & $24(48,0)$ & $40(38,5)$ & \\
\hline \multicolumn{4}{|l|}{ PANSS elevada } \\
\hline Sim & $13(26,0)$ & $27(26,0)$ & \multirow{3}{*}{0,463} \\
\hline Não & $32(64,0)$ & $72(69,2)$ & \\
\hline N.A. & $5(10,0)$ & $5(4,8)$ & \\
\hline \multicolumn{4}{|l|}{ Substâncias Psicoativas } \\
\hline Sim & $18(36,0)$ & $51(49,0)$ & \multirow{2}{*}{0,128} \\
\hline Não & $32(64,0)$ & $53(51,0)$ & \\
\hline \multicolumn{4}{|l|}{ Tabaco } \\
\hline Sim & $13(26,0)$ & $28(26,9)$ & \multirow{2}{*}{0,903} \\
\hline Não & $37(74,0)$ & $76(73,1)$ & \\
\hline \multicolumn{4}{|l|}{ Álcool } \\
\hline Sim & $13(26,0)$ & $38(36,5)$ & \multirow{2}{*}{0,193} \\
\hline Não & $37(74,0)$ & $66(63,5)$ & \\
\hline \multicolumn{4}{|l|}{ Maconha } \\
\hline Sim & $3(6,0)$ & $3(2,9)$ & \multirow{2}{*}{0,350} \\
\hline Não & $47(94,0)$ & $101(97,1)$ & \\
\hline \multicolumn{4}{|l|}{ Crack } \\
\hline Sim & $0(0,0)$ & $1(1,0)$ & \multirow{2}{*}{0,487} \\
\hline Não & $50(100,0)$ & $103(99,0)$ & \\
\hline \multicolumn{4}{|l|}{ Cocaína } \\
\hline $\operatorname{Sim}$ & $2(4,0)$ & $2(1,9)$ & \multirow{2}{*}{0,448} \\
\hline Não & $48(96,0)$ & $102(98,1)$ & \\
\hline \multicolumn{4}{|l|}{ Zabumba } \\
\hline Sim & $0(0,0)$ & $4(3,8)$ & \multirow{2}{*}{0,160} \\
\hline Não & $50(100,0)$ & $100(96,2)$ & \\
\hline \multicolumn{4}{|l|}{ Santo Daime } \\
\hline Sim & $0(0,0)$ & $4(3,8)$ & \multirow{2}{*}{0,160} \\
\hline Não & $50(100,0)$ & $100(96,2)$ & \\
\hline \multicolumn{4}{|l|}{ Uso de antipsicótico } \\
\hline Sim & $48(96,0)$ & $100(96,2)$ & 0,963 \\
\hline Não & $2(4,0)$ & $4(3,8)$ & 0,963 \\
\hline Uso de antipsicóticos típico & & & \\
\hline Sim & $37(74,0)$ & $75(72,1)$ & 0.806 \\
\hline Não & $13(26,0)$ & $29(27,9)$ & 0,000 \\
\hline Uso de antipsicótico atípico & & & \\
\hline Sim & $47(94,0)$ & $102(98,1)$ & 0181 \\
\hline Não & $3(6,0)$ & $2(1,9)$ & 0,101 \\
\hline Uso de antidepressivos & & & \\
\hline Sim & $23(46,0)$ & $55(52,9)$ & 0424 \\
\hline Não & $27(54,0)$ & $49(47,1)$ & $0,4 \angle 4$ \\
\hline
\end{tabular}

Legenda: Teste Qui-quadrado; IMC: Índice de Massa Corporal; PANSS: Escala de Avaliação da Síndrome Positiva e Negativa; NA: Não Avaliado; ${ }^{*} \mathrm{p}<0,05$. 
Tabela 2. Associação entre IMC e comorbidades em esquizofrênicos atendidos em um ambulatório especializado, entre os anos de 2011 a 2017, Fortaleza, Ceará.

\begin{tabular}{|c|c|c|c|c|c|c|}
\hline \multirow{3}{*}{ Características } & \multicolumn{2}{|c|}{$\operatorname{IMC}(n / \%)$} & \multirow{3}{*}{ p valor } & \multicolumn{3}{|c|}{ Regressão Logística Multinominal } \\
\hline & \multirow{2}{*}{$\begin{array}{l}<\mathbf{2 5} \mathbf{K g} / \mathbf{m}^{2} \\
\mathrm{n}=50(32,5)\end{array}$} & \multirow{2}{*}{$\begin{array}{c}\geq \mathbf{2 5 K g} / \mathbf{m}^{\mathbf{2}} \\
\mathrm{n}=104(67,5)\end{array}$} & & \multirow[t]{2}{*}{ OR } & \multicolumn{2}{|c|}{ IC } \\
\hline & & & & & Menor & Maior \\
\hline \multicolumn{7}{|l|}{ Comorbidades } \\
\hline Sim & $7(14,0)$ & $39(37,5)$ & \multirow{2}{*}{$0,003 *$} & \multirow[t]{2}{*}{3,686} & \multirow[t]{2}{*}{1,510} & \multirow[t]{2}{*}{8,993} \\
\hline Não & $43(86,0)$ & $65(62,5)$ & & & & \\
\hline \multicolumn{7}{|l|}{ Diabetes } \\
\hline Sim & $0(0,0)$ & $10(9,6)$ & \multirow{2}{*}{$0,023 *$} & \multirow[t]{2}{*}{1,106} & \multirow[t]{2}{*}{1,039} & \multirow[t]{2}{*}{1,178} \\
\hline Não & $50(100)$ & $94(90,4)$ & & & & \\
\hline \multicolumn{7}{|l|}{ Dislipidemia } \\
\hline Sim & $4(8,0)$ & $23(22,1)$ & \multirow{2}{*}{$0,031 *$} & \multirow[t]{2}{*}{3,265} & \multirow[t]{2}{*}{1,064} & \multirow[t]{2}{*}{10,026} \\
\hline Não & $46(92,0)$ & $81(77,9)$ & & & & \\
\hline \multicolumn{7}{|l|}{ Hipertensão } \\
\hline Sim & $2(4,0)$ & $15(14,4)$ & \multirow{2}{*}{0,053} & \multirow[t]{2}{*}{4,045} & \multirow[t]{2}{*}{0,888} & \multirow[t]{2}{*}{18,431} \\
\hline Não & $48(96,0)$ & $89(85,6)$ & & & & \\
\hline \multicolumn{7}{|l|}{ Hipotireoidismo } \\
\hline Sim & $2(40,0)$ & $5(4,8)$ & \multirow{2}{*}{0,822} & \multirow[t]{2}{*}{1,212} & \multirow[t]{2}{*}{0,227} & \multirow[t]{2}{*}{6,476} \\
\hline Não & $48(96,0)$ & $99(95,2)$ & & & & \\
\hline
\end{tabular}

Legenda: Teste Qui-quadrado; Regressão Logística Multinominal; IMC: Índice de Massa Corporal; IC: Intervalo de Confiança; OR: Odds Ratio; ${ }^{*} \mathrm{p}<0,05$.

\section{DISCUSSÃO}

A média de idade dos pacientes foi de 44,29 $\pm 14,32$ anos e com maior prevalência do sexo masculino $(58,4 \%)$. Estudos apontam que a prevalência entre sexos é equivalente, porém o início da doença ocorre de forma mais precoce em homens, além destes apresentam pior prognóstico do que as mulheres. Alguns autores associam isso ao fato de os homens geralmente demorem a buscar atendimento médico, possuírem maior tempo de internação e maior número de recaídas. ${ }^{6,7}$

Constatou-se que grande parte dos pacientes avaliados apresentavam excesso de peso $(65,58 \%) ; 31,81 \%$ estavam eutróficos e 2,59\% exibiam baixo peso. Resultados semelhantes foram encontrados em um estudo que avaliou o estado nutricional de 40 pacientes esquizofrênicos, em uso de antipsicóticos de segunda geração (ASG), atendidos por um Centro de atenção psicossocial (CAPS) em Porto Alegre e observaram que a maioria apresentava sobrepeso ou obesidade. ${ }^{4}$

Observou-se que 44,8\% dos pacientes avaliados já haviam feito uso de substâncias psicoativas. Alguns estudos mostram que o uso dessas substâncias pode ser predisponente ou agravante do prognóstico da esquizofrenia. ${ }^{8}$ Quanto ao uso de medicações, a maioria dos pacientes chegaram ao serviço utilizando ASG. Apesar de não ter sido encontrado associação entre o uso desses medicamentos com sobrepeso (IMC $\geq 25 \mathrm{~kg}$ / $\mathrm{m}^{2}$ ) em nossos resultados, há evidências na literatura de que os ASG são associados a efeitos colaterais indesejados ao metabolismo, como ganho de peso e riscos aumentado para o surgimento de comorbidades. ${ }^{9}$ Nesse sentido, um estudo feito no ano de 2012 mostrou a relação entre o uso de ASG com o ganho de peso e aparecimento de comorbidades, tendo como resultado o aumento do ganho de peso, colesterol e triglicerídeos em homens que faziam uso de olanzapina e o aumento de circunferência abdominal e glicemia em mulheres que usavam risperidona, o que suscita a necessidade de ter esses efeitos em conta durante o seguimento de pacientes em uso de ASG. ${ }^{10}$

Os resultados mostram que as comorbidades de maior prevalência na amostra estudada foram dislipidemia e hipertensão, que a longo prazo, corroboram para o desenvolvimento de doenças cardiovasculares, atualmente uma das principais causas de morte em pessoas com esquizofrenia. ${ }^{11}$ Ademais, quando comparada à população geral, a incidência de sobrepeso, dislipidemia e diabetes em esquizofrênicos é alta, como constatado por Leitão-Azevedo et al., ${ }^{12}$ que avaliou 124 pacientes diagnosticados com esquizofrenia. Os autores encontraram em sua população um índice de $84,7 \%$ de pacientes com dislipidemia e 40,3\% com alterações glicêmicas. Além disso, houve associação significativa entre IMC $\geq 25 \mathrm{~kg} / \mathrm{m}^{2}$ e comorbidades, como diabetes e dislipidemia. Em nosso estudo, a prevalência de comorbidades foi inferior a encontrada por Leitão-Azevedo et al., ${ }^{12}$ no entanto, ressalta-se que os pacientes foram 
avaliados ao diagnóstico de esquizofrenia. A predominância de sobrepeso, obesidade e comorbidades metabólicas como dislipidemia e HAS e a associação entre os esses fatores pode ser justificada devido às mudanças alimentares e fisiológicas que estes pacientes apresentam.

Não foram encontradas associações entre o IMC, a presença de comorbidades e o tipo de antipsicótico utilizado. Possivelmente, a maior prevalência de comorbidades clínicas em nossa amostra pode estar relacionada com variáveis comportamentais não avaliadas, porém, colocados como possíveis contribuintes em outros estudos, tais como o isolamento social e dificuldade de comunicação. ${ }^{13}$

Um achado importante nesse estudo foi a correlação negativa significativa entre a PANSS positiva e o IMC, mostrando a influência do aumento do grau de sintomas, como delírios e alucinações, na redução do IMC. A recusa alimentar e a perda de peso intencional ou não são sintomas frequentes entre portadores de esquizofrenia. Em um estado de psicose aguda, esses pacientes apresentaram redução do consumo alimentar e inapetência em meio a um surto psicótico. ${ }^{14,15}$ Tais publicações corroboram com os nossos achados. Melo et al., ${ }^{16}$ investigaram a associação entre PANSS negativa e IMC, porém, os autores não encontraram significância estatística. Este resultado assemelha-se ao observado em nossa pesquisa. Os sintomas positivos não foram investigados pelos autores.

\section{REFERÊNCIAS}

1. American Psychiatry Association. Diagnostic and Statistical Manual of Mental disorders - DSM-5. 5th.ed. Washington: American Psychiatric Association; 2013.

2. Vannice GK. Terapia Nutricional para Condições Psiquiatricas. In: Mahan L, Escott-Stump S, Raymond J. Krause: alimentos, nutrição e dietoterapia. 13th ed. Rio de Janeiro: Elsevier; 2013. Cap. 42, p. 956-971.

3. Franch Pato C, Molina Rodríguez V, Franch Valverde J. Síndrome metabólico y antipsicóticos atípicos. Posibilidad de predicción y control. Rev psiquiatr salud ment. 2017;10(1):38-44.

4. Zortéa K, Guimarães L, Gama C, Belmonte-de-Abreu P. Estado nutricional de pacientes com esquizofrenia frequentadores do Centro de Atenção Psicossocial (CAPS) do Hospital de Clínicas de Porto Alegre. J Bras de Psiquiatr. 2010;59(2):126-30.

5. Obermeier M. The Positive and Negative Syndrome Scale for Schizophrenia: An Established Rating Instrument in Need of Clarification [online]. Ludwig-Maximilians-Universität zu München; 2012. [Acessado 27 Janeiro 2017]. Disponível em: <https://edoc. ub.uni-muenchen.de/14936/>

6. Chaves AC. Diferenças entre os sexos na esquizofrenia. Rev Bras Psiquiatr. 2000;22(Supl 2): 21-2.

7. Nicolino PS, Vedana KG, Miasso AI, Cardoso L, Galera SA. Esquizofrenia: adesão ao tratamento e crenças sobre o transtorno e terapêutica medicamentosa. Rev Esc Enferm USP.2011;45(3):708-15.
Dentre as limitações do nosso estudo, pode-se citar a falta de informação nutricional anterior ao início do uso de ASG por uma parte dos pacientes investigados, visto que a maior parte desses indivíduos chegaram ao serviço especializado já em uso dessas substâncias. Alguns pacientes possuíam registros incompletos da PANSS na primeira consulta, impossibilitando essa avaliação. No entanto, destaca-se que esse é o primeiro estudo que correlacionou o grau de sintomas positivos, através da PANSS, que é uma ferramenta validada e amplamente utilizada na prática clínica, com o estado nutricional de portadores de esquizofrenia.

\section{CONCLUSÃO}

Foi possível notar que o agravamento dos sintomas positivos, a partir dos resultados apresentados da PANSS, pode ter influência na diminuição do peso corporal em pessoas com esquizofrenia. Quanto ao uso de medicamentos antipsicóticos, não foi encontrado, em nossa casuística, associações significativas com o estado nutricional. Embora existam muitos fatores que levem a essas condições, os resultados corroboram para a importância do cuidado nutricional em todas as fases do tratamento da doença, visto que pacientes esquizofrênicos, sejam por perda ou excesso de peso, caracterizam-se como um grupo de risco nutricional. Adicionalmente, mais estudos são necessários para melhor entender as relações entre a sintomatologia, o tratamento farmacológico e as mudanças no peso corporal nesse grupo.

8. Silveira J, Oliveira R, Viola B, Silva T, Machado R. Schizophrenia and the use of alcohol and other drugs: epidemiological profile. Revista da Rede de Enfermagem do Nordeste. 2014;15(3):43646.

9. Schmitz A, Kreutz O, Suyenaga E. Antipsicóticos Atípicos Versus Efeito Obesogênico Sob A Óptica Da Química Medicinal. Revista Eletrônica de Farmácia. 2015;12(3):23.

10. Chaves KM, Ribeiro SB, Lima BLS, Guerra GC, Soares LA, Araújo AA. Alterações antropométricas e bioquímicas em usuários crônicos dos antipsicóticos olanzapina e risperidona. Rev Bras Farm. 2012;93(3):359-63.

11. Azad MC, Shoesmith WD, Al Mamun M, Abdullah AF, Naing DKS, Phanindranath $\mathrm{M}$ et al. Cardiovascular diseases among patients with schizophenia. Asian J Psychiatr. 2016;19:28-36.

12. Leitão-Azevedo CL, Guimarães LR, Abreu MGB, Gama CS, Lobato MI, Belmonte-de-Abreu PS. Increased dyslipidemia in schizophrenic outpatients using new generation antipsychotics. Rev Bras Psiquiatr. 2006;28(4):301-4.

13. Nishanth KN, Chadda RK, Sood M, Biswas A, Lakshmy R. Physical comorbidity in schizophrenia \& its correlates. Indian Indian J Med Res. 2017;146(2):281-4.

14. Sadock BJ, Sadock VA, Ruiz P. Transtornos do Espectro da Esquizofrenia e Outros Transtornos psicóticos. In: Sadock BJ, Sadock VA, Ruiz P. Compêndio de Psiquiatria: Ciência do Comportamento e 
Psiquiatria Clínica. 11.ed. Porto Alegre: Artmed Editora; 2017. Cap. 07, p. 300-46.

15. Teixeira EH, Meneguette J, Dalgalarrondo P. Matricídio, seguido de canibalismo e automutilação de pênis e mão em paciente esquizofrênico motivado por delírios religiosos. J Bras de Psiquiatr.
2012;61(3):185-8.

16. Melo MC, Albuquerque SG, Luz JH, Quental PT, Sampaio AM, Lima AB. Perfil clínico e psicossocial dos moradores em hospitais psiquiátricos no estado do Ceará, Brasil. Ciência \& Saúde Coletiva. 2015:20(2):343-52.

\section{Como citar:}

Ricarte JR, Recamonde JM, Pitts NF, Aguiar AP, Lima RS, Souza FG, et al. Relação entre índice de massa corporal e escala de avaliação da síndrome positiva e negativa em portadores de esquizofrenia atendidos em um serviço especializado. Rev Med UFC. 2022;62(1):1-6. 